\title{
EFEITOS DE POLÍMEROS HIDRORRETENTORES NAS PROPRIEDADES FÍSICO-HÍDRICAS DE DOIS MEIOS POROSOS ${ }^{(1)}$
}

\author{
C. L. PREVEDELLO ${ }^{(2)} \&$ S. P. BALENA ${ }^{(3)}$
}

\begin{abstract}
RESUMO
Com o propósito de avaliar os efeitos de polímeros hidrorretentores nas propriedades físicas e hidráulicas de dois meios porosos, realizou-se um experimento no Laboratório de Física do Solo da Universidade Federal do Paraná, entre 18/03 e 30/10/97. O polímero hidrorretentor usado foi produzido na Bél gica e os meios porosos foram um Latossolo Vermelho textura argilosa e uma Areia Quartzosa Marinha, ambos na forma de TFSA. Os polímeros foram aplicados na forma de grãos passados em peneira de 0,5 e $1 \mathrm{~mm}$ de diâmetro, nas seguintes concentrações: $0,2,4,8,16$ e $32 \mathrm{~kg} \mathrm{~m}^{-3}$. Foram elaboradas as curvas de retenção a baixas tensões $\left(0 ; 0,025 ; 0,045 ; 0,10 ; 0,20 ; 0,60 ; 1,5\right.$ e $\left.3,0 \mathrm{mH}_{2} \mathrm{O}\right)$, medidas as condutividades hidráulicas saturadas e esti mados os diâmetros médi os de poros. O processo da evaporação de água do solo foi si mulado por modelagem numérica. As curvas de retenção de água medidas e os perfis de umidade simulados da evaporação afastaram-se consideravel mente da origem (testemunhas) pela adi ção de polímeros, particularmente na Areia Quartzosa Marinha. 0 diâmetro médio de poros também aumentou progressivamente com o aumento da concentração de polímeros. Foi verificado que, nas concentrações de polímeros acima de $8 \mathrm{~kg} \mathrm{~m}^{-3}$, as propriedades físico-hídricas dos meios porosos foram dominadas pela ação dos polímeros hidrorretentores.
\end{abstract}

Termos de indexação: polímeros hidrorretentores, hidrogéis, retenção de água.

(1) Trabal ho enviado para publicação em agosto de 1999 e aprovado em março de 2000.

(2) Professor Titular do Departamento de Solos, UniversidadeF ederal do Paraná - UFPR. Caixa Postal 2959, CEP 80035-050 Curitiba (PR). E-mail: cl preve@agrarias.ufpr.br

(3) Física, MSc. em Agronomia, CEFET-PR/UNED-PB, Caixa Postal 571, CEP 85503-390 Pato Branco (PR). 


\begin{abstract}
EFFECT OF HYDRORETENTIVE POLYMERS ON THE PHYSICAL AND HYDRAULIC PROPERTIES OF TWO POROUS MEDIA
\end{abstract}

\begin{abstract}
Thepurpose of this work was to verify the effect of hidroretentivepolymers on thephysical and hydraulic properties of two porous media. Theexperiments were carried out at the Soil Physics Laboratory at theF ederal University of Paraná from 03/ 18 to 10/30/97. Theresearch was devel oped using a Bel gi um type of retentive pol ymer incorporated in two porous media with their different physico-chemical properties; a Dark Red Oxisol and MarineQuartzous sand. The pol ymers wereapplied as dried grains at the foll owing concentrations: $0,2,4,8$, 16 and $32 \mathrm{~kg} \mathrm{~m}^{-3}$. Water retention curves were elaborated for the tensions of $0.00 ; 0.025$; $0.045 ; 0.10 ; 0.20 ; 0.60 ; 1.50$ and $3.00 \mathrm{mH}_{2} \mathrm{O}$. The values of the saturated hydraulic conductivity were measured and the mean diameter of the pores estimated. The process of water evaporation from soil was considered for numeric simulation. Water retenti on curves and themoistureprofiles by simulated evaporation wereconsiderably different from reference points after theaddition of polymers, particul arly in theM arineQuartzous sand. Themean diameter of pores progressively increased with increasing polymer concentration. It was al so verified that at the concentrations of $8 \mathrm{~kg} \mathrm{~m}^{-3}$, the physical and hydraulic properties of thesoil did not present their natural effects and wereovercomeby theaction of the polymers.
\end{abstract}

Index terms: hydroretentive polymers, hydrogels, water retention.

\section{NTRODUÇÃO}

A necessidade de aumentar a produção de alimentos, aliada à globalização do comércio e à competitividade dos produtos agrícolas, tem estimulado cooperativas e produtores a buscarem técnicas alternativas para a melhoria da produtividade e redução de custos. Deste modo, os polímeros hidrorretentores passaram a ser pesquisados como forma de minimizar os problemas associados à baixa produtividade, geralmente provocada pela disponibilidade irregular ou deficitária deágua e má estruturação do sol o. E sses polímeros, arranjo de moléculas orgânicas, quando secos, apresentam forma granular e quebradiça. Ao serem hidratados, transformam-se em gel, cuja forma macia e elástica possibilita absorver cerca de cem vezes o seu peso em água, ou mais (F onteno \& Bilderback, 1993). I nicialmente utilizados como alternativa de produção para as regiões de clima árido, sua aplicação tornou-se conhecida nas diferentes partes do planeta, diversificando-se, sobremaneira, em hortaliças e culturas anuais. A maior parte das pesquisas realizadas mostrou-se favorável ao emprego de polímeros nos solos agrícolas, apresentando como principal fator de convergência a melhor utilização da água (Flannery \& Busscher, 1982; Azzam, 1983; Baasiri et al., 1986; Blodgett et al., 1993; Volkmar \& Chang, 1995).

O objetivo deste trabal ho foi avaliar os efeitos de polímer os hidrorretentores nas propriedades físicas e hidráulicas de dois meios porosos de distintas propriedades físico-químicas.

\section{MATERIAL E MÉTODOS}

Foram usadas diferentes concentrações de um tipo de polímero hidrorretentor industrializado na Bélgica, disponibilizado no Brasil pela Terracotem do Brasil Ltda., em dois meios porosos de distintas propriedades físico-químicas: um L atossol oVermelho textura argilosa, do município de Pato Branco (PR), e uma Areia Quartzosa Marinha, do balneário Caiobá, município de Matinhos, litoral paranaense. O polímero utilizado continha 49,75\% desílica, 39,5\% demoléculas orgânicas, 1,05\% de macroelementos e $0,25 \%$ de estimulantes de crescimento. As concentrações de polímero usadas foram: $0 ; 2 ; 4 ; 8$; 16 e $32 \mathrm{~kg} \mathrm{~m}^{-3}$. Os materiais porosos foram utilizados na forma de TFSA, acondicionados em cilindros de PVC, com $40 \mathrm{~mm}$ de diâmetro e $30 \mathrm{~mm}$ de altura.

Os ensaios foram realizados no laboratório de Física do Solo da UFPR. Para que a incorporação desses polímeros fosse mais homogênea, considerando as reduzidas concentrações e dimensões das amostras, além da grande variabilidade granulomé trica do polímero usado, optou-se por peneirá-lo, aproveitando somente as frações retidas entre as malhas de 0,5 e 1,0 mm de diâmetro. Testes prévios mostraram que esse procedimento não alterou as propriedades de retenção do polímero.

Foram el aboradas as curvas de retenção deágua, com cinco repetições por concentração de polímeroe para cada meio por oso. As tensões utilizadas foram: $0 ; 0,025 ; 0,045 ; 0,10 ; 0,20 ; 0,60 ; 1,50$ e $3,00 \mathrm{mH}_{2} \mathrm{O}$. A condutividade hidráulica saturada, $\mathrm{K}_{s}$, foi 
determinada por meio de permeâmetro de carga constante (média de cinco repetições). Utilizando a equação de Van Genuchten (1980), qual seja,

$$
\theta(|\mathrm{p} / \rho \mathrm{g}|)=\theta_{\mathrm{r}}+\frac{\left(\theta_{\mathrm{s}}-\theta_{\mathrm{r}}\right)}{\left[1+(\alpha|\mathrm{p} / \rho \mathrm{gg}|)^{\mathrm{n}}\right]^{\mathrm{m}}}
$$

os dados médios da curva de retenção foram ajustados por regressão não-linear, conforme Boratto (1984), conhecendo-se, desta forma, os parâmetros independentes $\theta_{r}, \theta_{s}, \alpha$, men, da equação (1), os quais, juntamente com os val ores de $K_{s}$, foram usados para calcular a condutividade hidráulica em função da umidade pela expressão (Van Genuchten, 1980):

$$
\mathrm{K}(\theta)=\mathrm{K}_{\mathrm{s}}\left[\frac{\theta-\theta_{\mathrm{r}}}{\theta_{\mathrm{s}}-\theta_{\mathrm{r}}}\right]^{1 / 2}\left\{1-\left[1-\left(\frac{\theta-\theta_{\mathrm{r}}}{\theta_{\mathrm{s}}-\theta_{\mathrm{r}}}\right)^{1 / \mathrm{m}}\right]^{\mathrm{m}}\right\}^{2}
$$

Com o auxílio da expressão definida por Prevedello (1996), qual seja,

$$
\mathrm{r}=\frac{2 \sigma \alpha \cos \phi}{\rho \mathrm{g}}\left[\frac{\mathrm{n}-1}{\mathrm{n}}\right]^{-1 / \mathrm{n}}
$$

calcul ou-se otamanho de poros que continham maior quantidade deágua num solo (que armazenam mais água), convencional mente chamado “tamanho médio de poros".

De posse dos parâmetros independentes da equação (1), juntamente com os valores da condutividade hidráulica saturada, e do modelo numérico proposto por Prevedello (1996) para a simulação do processo da evaporação da água no solo, realizou-se a simulação da evaporação de água e dos perfis de umi dade após $168 \mathrm{~h}$ de evaporação, na taxa de $5 \mathrm{~mm} / \mathrm{d}$, para as diferentes concentrações de polímero, nos dois meios porosos.

Finalmente, visando identificar a concentração a partir da qual os polímeros exercem influência dominante sobre a natureza dos meios porosos, estabeleceram-se, entre o Latossolo e a Areia Quartzosa Marinha, as relações dos valores de umi dade nas diversas tensões $1 \mathrm{p} / \mathrm{\rho g}$, dos parâmetros independentes da equação (1) e do diâmetro de poros que armazenam mais água (E quação 3).

\section{RESULTADOS E DISCUSSÃO}

\section{E feito dos polímeros nas propriedades físico- hídricas}

Os polímeros produziram um decréscimo nos valores da condutividade hidráulica saturada $\left(\mathrm{K}_{\mathrm{s}}\right)$, tanto no Latossolo quanto na Areia Quartzosa Marinha. No Latossolo, o decréscimo ocorreu de forma gradual, embora sua grande expressividade tenha sido para a concentração de $32 \mathrm{~kg} \mathrm{~m}^{-3}$. Na Areia Quartzosa Marinha, os valores do $K_{s}$ apresentaram pequenas variações atéà concentração de $8 \mathrm{~kg} \mathrm{~m}^{-3}$, decrescendo proporcional-mente com o aumento da concentração de polímeros, tornando-se aproximadamente cinco vezes menor para a concentração de $32 \mathrm{~kg} \mathrm{~m}^{-3}$. O quadro 1 mostra os valores da condutividade hidráulica saturada $\left(\mathrm{K}_{\mathrm{s}}\right)$, por concentração de polímero, para os dois mei os porosos.

Quanto maior a concentração de polímeros, maiores foram os valores dos parâmetros $\theta_{s,}, \theta_{r}, \alpha$, tanto para o Latossolo quanto para a Áreia Quartzosa Marinha, como pode ser observado no quadro 2, para o Latossolo, e no quadro 3, para a Areia Quartzosa Marinha.

As figuras 1A e 1B mostram as curvas de retenção deágua para o Latossolo eAreia Quartzosa Marinha, respectivamente. Nota-se que a adição de polímeros aumentou a retenção deágua nos dois meios porosos. No L atossolo, a capacidade de retenção deágua para a concentração de $32 \mathrm{~kg} \mathrm{~m}^{-3}$ foi acrescida em 1,3 vez para a menor tensão $\left(0,025 \mathrm{mH}_{2} \mathrm{O}\right)$ e 1,9 vez para a maior tensão $\left(3,00 \mathrm{mH}_{2} \mathrm{O}\right)$. O incremento foi ainda

\section{Quadro 1. Valores médios (média de cinco repetições) da conduti vidade hidráulica saturada $\left(K_{s}\right)$, por

\begin{tabular}{|c|c|c|}
\hline $\begin{array}{l}\text { Concentração } \\
\text { de polímero }\end{array}$ & $\begin{array}{c}\mathrm{K}_{\mathrm{s}} \\
\text { do Latossolo }\end{array}$ & $\begin{array}{c}\mathrm{K}_{\mathbf{s}} \\
\text { da Areia Marinha }\end{array}$ \\
\hline $\mathrm{kg} \mathrm{m}^{-3}$ & \multicolumn{2}{|c|}{$\longrightarrow \mathrm{m} \mathrm{s}^{-1}$} \\
\hline 0 & $4,38 \times 10^{-4}$ & $1,63 \times 10^{-4}$ \\
\hline 2 & $3,05 \times 10^{-4}$ & $1,37 \times 10^{-4}$ \\
\hline 4 & $2,34 \times 10^{-4}$ & $1,16 \times 10^{-4}$ \\
\hline 8 & $2,25 \times 10^{-4}$ & $0,59 \times 10^{-4}$ \\
\hline 16 & $2,13 \times 10^{-4}$ & $0,46 \times 10^{-4}$ \\
\hline 32 & $1,03 \times 10^{-4}$ & $0,34 \times 10^{-4}$ \\
\hline
\end{tabular} concentração de polímero, para os dois meios porosos}

Quadro 2. Parâmetros da equação de Van Genuchten (1980) para o Latossolo

\begin{tabular}{ccccccc}
\hline Concentração & $\alpha$ & $\mathbf{m}$ & $\mathbf{n}$ & $\theta_{\mathbf{r}}$ & $\theta_{\mathbf{s}}$ & $\mathbf{r}^{\mathbf{2}}$ \\
\hline $\mathrm{kg} \mathrm{m}^{-3}$ & $\mathrm{~m}^{-1}$ & & \multicolumn{4}{c}{$-\mathrm{m}^{3} \mathrm{~m}^{-3}-$} \\
0 & 3,824 & 0,747 & 3,986 & 0,278 & 0,737 & 1,000 \\
2 & 4,489 & 0,652 & 2,872 & 0,306 & 0,773 & 0,998 \\
4 & 3,749 & 0,632 & 2,713 & 0,334 & 0,860 & 0,995 \\
8 & 6,990 & 0,567 & 2,311 & 0,354 & 0,878 & 0,999 \\
16 & 8,343 & 0,556 & 2,250 & 0,418 & 0,903 & 0,995 \\
32 & 8,909 & 0,535 & 2,149 & 0,508 & 0,941 & 0,996 \\
& & & & & &
\end{tabular}


Quadro 3. Parâmetros da equação de Van Genuchten (1980) para a Areia Quartzosa Marinha

\begin{tabular}{ccccccc}
\hline Concentração & $\alpha$ & $\mathbf{m}$ & $\mathbf{n}$ & $\boldsymbol{\theta}_{\mathbf{r}}$ & $\boldsymbol{\theta}_{\mathbf{s}}$ & $\mathbf{r}^{\mathbf{2}}$ \\
\hline $\mathrm{kg} \mathrm{m}^{-3}$ & $\mathrm{~m}^{-1}$ & & \multicolumn{4}{c}{$-\mathrm{m}^{3} \mathrm{~m}^{-3}-$} \\
0 & 2,528 & 0,628 & 2,688 & 0,046 & 0,447 & 0,998 \\
2 & 1,543 & 0,764 & 4,233 & 0,059 & 0,509 & 0,998 \\
4 & 1,922 & 0,694 & 3,267 & 0,118 & 0,589 & 0,998 \\
8 & 6,990 & 0,563 & 2,286 & 0,338 & 0,650 & 0,999 \\
16 & 9,354 & 0,593 & 2,459 & 0,368 & 0,783 & 0,998 \\
32 & 9,977 & 0,580 & 2,383 & 0,459 & 0,922 & 0,999 \\
\hline
\end{tabular}

mai or na Areia Quartzosa Marinha, registrando um aumento duas vezes maior para a menor tensão e 7,5 vezes maior para a mai or tensão. Comparandoa inclinação das curvas de retenção de ambos os meios, observa-se que elas adquirem forma muito semel hantes, a partir da concentração de $8 \mathrm{~kg} \mathrm{~m}^{-3}$.
De posse dos valores de $\theta_{r}, \theta_{s}, \alpha, n$, me $_{s}$, foram calculadas, pela equação (2), as funções $K(\theta)$ e elaboradas as curvas das figuras $2 \mathrm{~A}, \ldots 2 \mathrm{~F}$, que representam a condutividade hidráulica em função da umidade volumétrica, para ambos os meios porosos.

É interessante observar, nessas figuras, que, com o aumento da concentração de polímero, as curvas da Areia Quartzosa Marinha aproximam-se gradativamente das do Latossolo, sobrepondo-se a partir da concentração de $16 \mathrm{~kg} \mathrm{~m}^{-3}$, como se, a partir dessa concentração, a função $K(\theta)$ fosse unicamente influenciada pela ação do polímero. I sso demonstra, com efeito, que a ação do polímero sobre a retenção de água não foi igual para os dois meios porosos, sendo maior para a Areia Quartzosa Marinha do que para o L atossolo.

\section{Efeito dos polímeros no tamanho médio de poros}

O quadro 4 apresenta os valores dos diâmetros médi os de poros (DMP) nas diferentes concentrações de polímeros, para os dois meios porosos, segundo Prevedello (1996). O DMP, segundo esse autor,

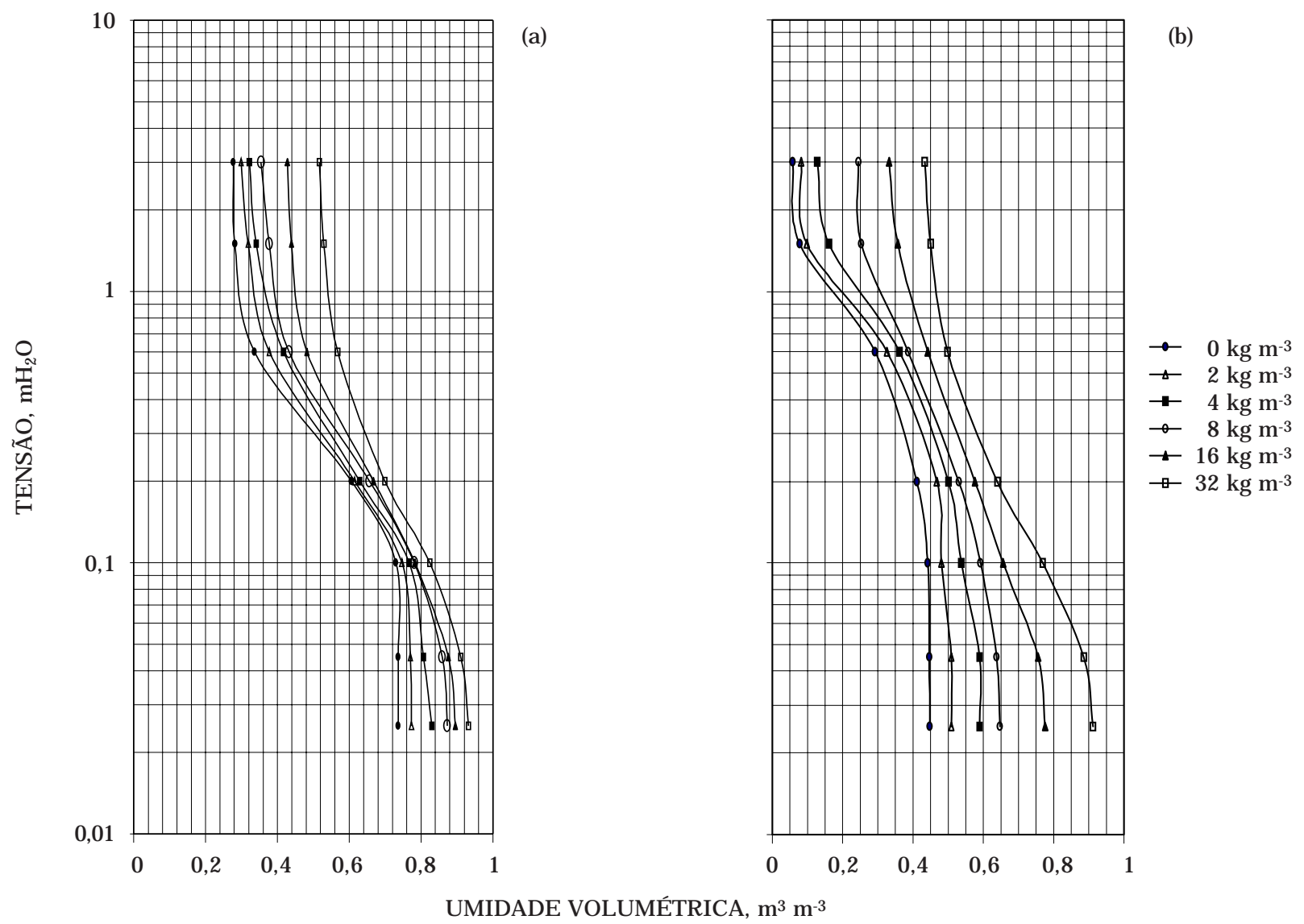

Figura 1. Curva de retenção de água para o Latossolo (a) e Areia Quartzosa Marinha (b), para diversas concentrações de polímero. 


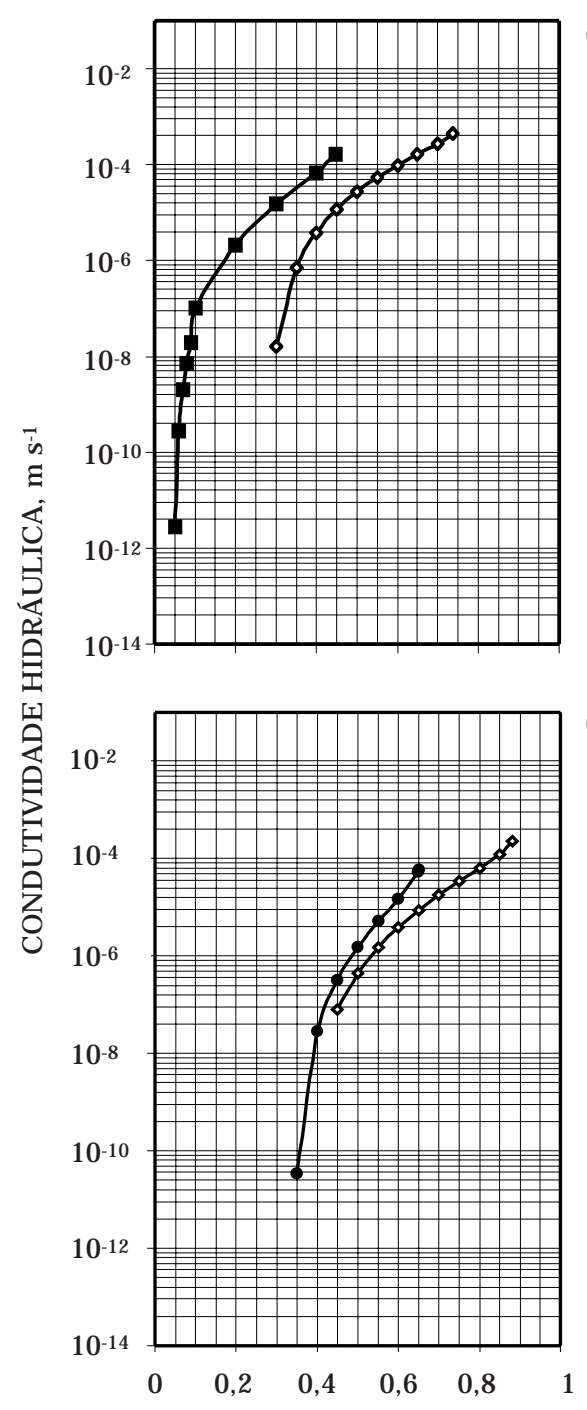

(a)

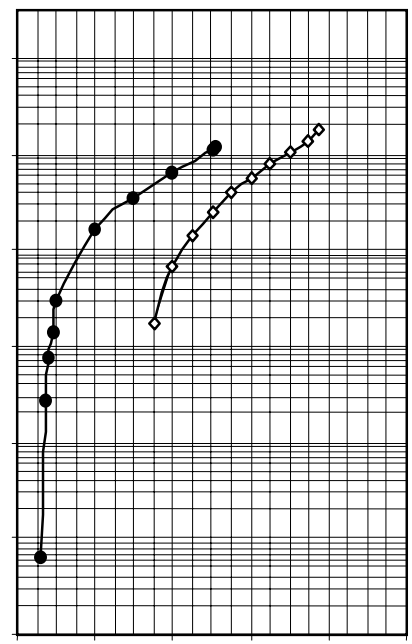

(b)

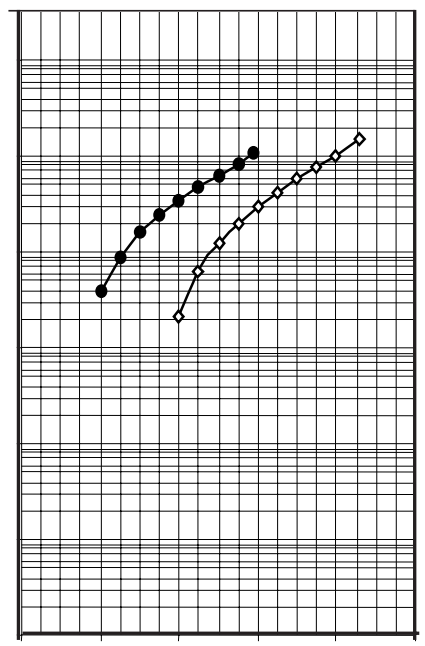

(d)

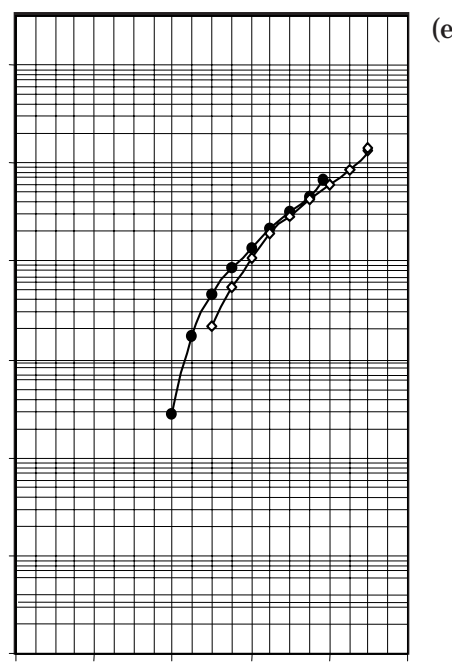

(e)

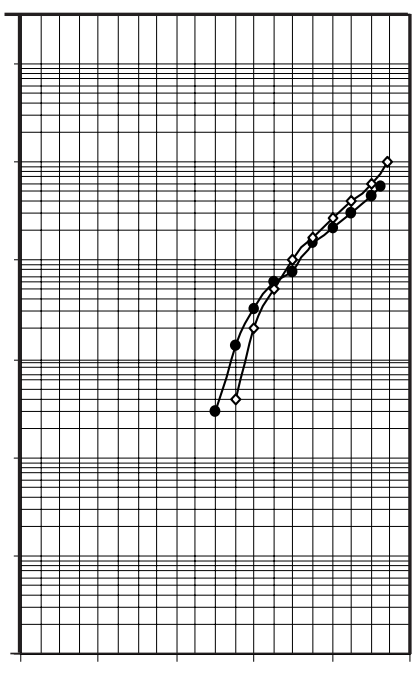

$\begin{array}{llllll}0 & 0,2 & 0,4 & 0,6 & 0,8 & 1\end{array}$

(c)

(f)

UMIDADE VOLUMÉTRICA, $\mathrm{m}^{3} \mathrm{~m}^{-3}$

Figura 2. Condutividade hidráulica em função da umidade para as concentrações de hidrorretentor: $a=0$; $b=2 ; c=4 ; d=8 ; e=16 ; f=32 k g ~ m^{-3}$. Símbolos cheios: Latossolo; símbolos vazios: Areia Quartzosa Marinha.

representa o diâmetro de poros quearmazenam mais água. Em seu estado natural, oDMP foi 1,4 vez maior para o Latossolo do que para a Areia Quartzosa Marinha. Com a adição do polímero, sofreu poucas alterações atéà concentração de $8 \mathrm{~kg} \mathrm{~m}^{-3}$. A partir daí, os diâmetros de poros que armazenaram mais água tiveram aumentos progressivos nos dois meios porosos. No Latossolo, o aumento em relação à testemunha foi de 2,20; 2,64 e 2,91, para as concentrações respectivas de 8; 16 e $32 \mathrm{~kg} \mathrm{~m}^{-3}$; e na Areia, de 3,00; 3,85 e 4,20, para as mesmas concentrações.

\section{Efeito dos polímeros na conservação da água do solo}

A seqüência dos experimentos revelou que, quanto maior a concentração de polímeros, maiores
Quadro 4. Diâmetro médio de poros que armazenam mais água para o Latossolo e para a Areia Quartzosa Marinha

\begin{tabular}{ccc}
\hline \multirow{2}{*}{$\begin{array}{c}\text { Concentração } \\
\text { de polímero }\end{array}$} & Diâmetro médio de poros \\
\cline { 2 - 3 } & Latossolo & $\begin{array}{c}\text { Areia Quartzosa } \\
\text { Marinha }\end{array}$ \\
\hline $\mathrm{kg} \mathrm{m}^{-3}$ & 122,0 & \\
0 & 155,0 & 89,4 \\
2 & 132,0 & 48,8 \\
4 & 265,8 & 63,8 \\
8 & 322,0 & 267,4 \\
16 & 354,8 & 344,0 \\
32 & & 373,2 \\
\hline
\end{tabular}


Quadro 5. Razão adimensional para p/pg do Latossolo e da Areia Quartzosa Marinha

\begin{tabular}{cccccccc}
\hline \multirow{2}{*}{ Concentração } & \multicolumn{7}{c}{ Tensão $\left(\mathbf{m H}_{\mathbf{2}} \mathbf{O}\right)$} \\
\cline { 2 - 8 } & $\mathbf{2 , 5}$ & $\mathbf{4 , 5}$ & $\mathbf{1 0}$ & $\mathbf{2 0}$ & $\mathbf{6 0}$ & $\mathbf{1 5 0}$ & $\mathbf{3 0 0}$ \\
\hline 0 & 1,649 & 1,643 & 1,339 & 1,622 & 1,425 & 3,5 & 6,043 \\
2 & 1,447 & 1,458 & 1,450 & 1,353 & 1,181 & 3,6 & 5,186 \\
4 & 1,465 & 1,497 & 1,395 & 1,351 & 1,236 & 2,34 & 2,831 \\
8 & 1,351 & 1,350 & 1,315 & 1,254 & 1,105 & 1,092 & 1,047 \\
16 & 1,153 & 1,133 & 1,182 & 1,268 & 1,153 & 1,176 & 1,136 \\
32 & 1,021 & 1,041 & 1,058 & 1,148 & 1,137 & 1,128 & 1,107 \\
\hline
\end{tabular}

Quadro 6. Razão adimensional dos parâmetros independentes: $\theta_{r}, \theta_{s}, \alpha, m, n$; e do DMP entre o Latossolo e a Areia Quartzosa Marinha

\begin{tabular}{ccccccc}
\hline Concentração & \multicolumn{1}{c}{$\boldsymbol{\alpha}$} & $\mathbf{m}$ & $\mathbf{n}$ & $\boldsymbol{\theta}_{\mathbf{r}}$ & $\boldsymbol{\theta}_{\mathbf{s}}$ & DMP \\
\hline 0 & 1,513 & 1,189 & 1,483 & 6,043 & 1,649 & 1,365 \\
2 & 2,909 & 0,853 & 0,678 & 5,186 & 1,447 & 3,176 \\
4 & 1,951 & 0,911 & 0,830 & 2,831 & 1,465 & 2,069 \\
8 & 1,00 & 1,007 & 1,011 & 1,047 & 1,351 & 0,994 \\
16 & 0,892 & 0,938 & 0,915 & 1,136 & 1,153 & 0,936 \\
32 & 0,893 & 0,922 & 0,902 & 1,107 & 1,021 & 0,951 \\
\hline
\end{tabular}

UMIDADE VOLUMÉTRICA, $\mathrm{m}^{3} \mathrm{~m}^{-3}$
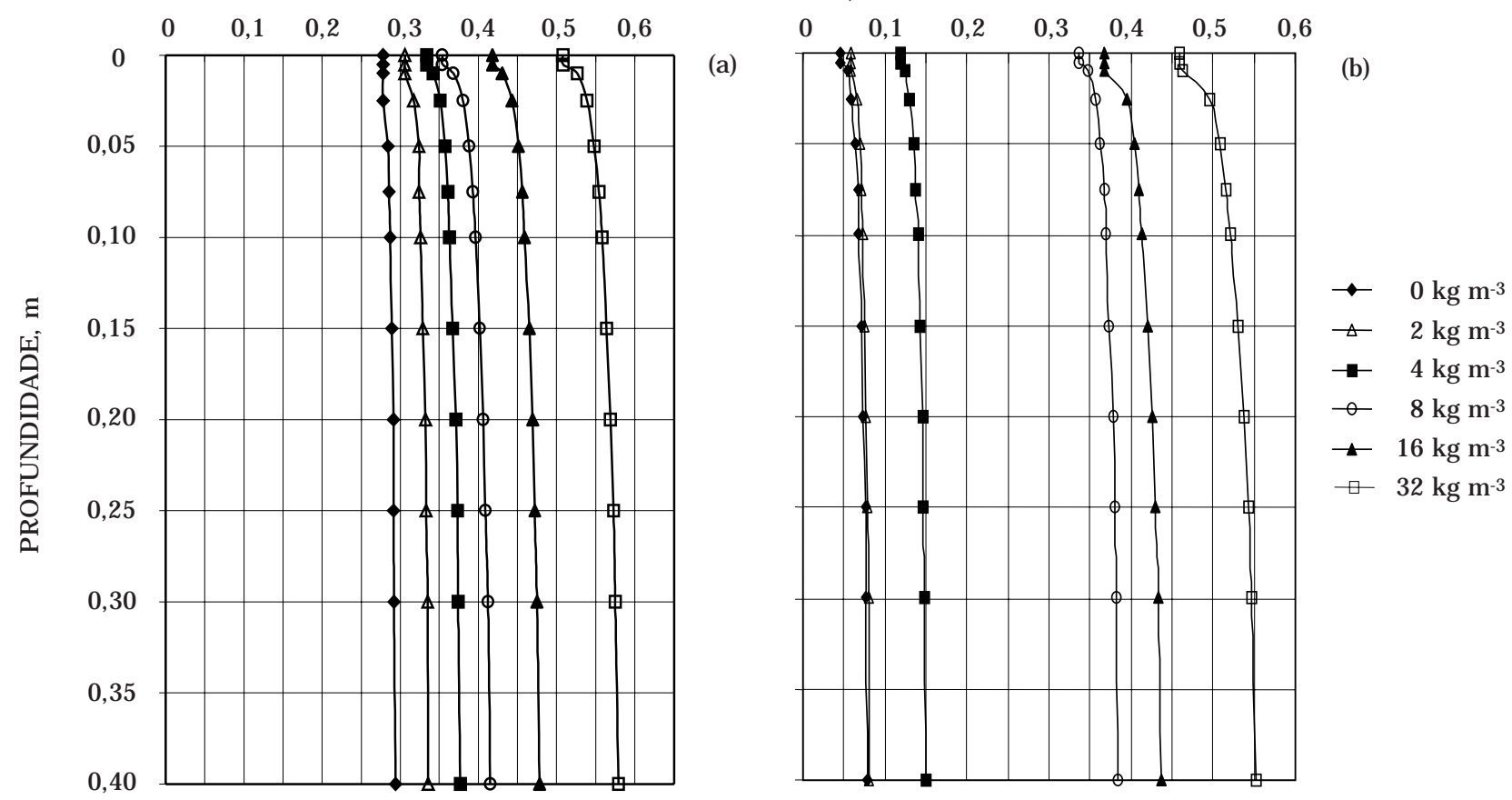

Figura 3. Perfis de umidade si mulados pelo modelo numérico de Prevedello (1996) para o Latossolo (a) e Areia Quartzosa Marinha (b). 
foram os val ores dos parâmetros $\theta_{r}, \theta_{s}$ e $\alpha$ e menores os valores do $K_{s}$, nos dois meios porosos. Como conseqüência, os perfis de umidade desses meios, para qualquer demanda evaporativa simulada, após $168 \mathrm{~h}$ de evaporação, na taxa de $5 \mathrm{~mm} / \mathrm{d}$, a partir de uma umidade inicial correspondente à tensão de $0,01 \mathrm{mH}_{2} \mathrm{O}$, sempre se mostraram mais afastados da origem quanto maior a concentração de polímeros aplicada, como pode ser observado nas figuras $4 a$ e $4 b$.

A adição de polímeros aumentou progressivamente a umi dade do solo, chegando a duplicar a capacidade de armazenamento da água para a concentração de $32 \mathrm{~kg} \mathrm{~m}^{-3}$, o que mostra a grande capacidade desse material em reter e conservar água no solo por períodos apreciáveis de evaporação. Esse efeito é ainda mais evidentena Areia, ondeo armazenamento, para o mesmo período de tempo, taxa de evaporação e concentração de polímeros, foi aumentado em cerca de 7,5 vezes. Esses resultados concordam com as afirmações de Tayel et al. (1981) e Wang \& Gregg (1990), que dizem que os polímer os hidrorretentores podem ser considerados uma forma eficaz de reduzir a evaporação de água e melhorar o regime hídrico dos solos.

\section{Domínio dos polímeros sobre os efeitos naturais dos meios porosos}

Visando identificar a concentração a partir da qual os polímeros exercem influência dominante sobre a natureza dos meios porosos, foram divididos os val ores dos parâmetros $\theta_{r}, \theta_{s}, \alpha, m, n, D M P$ elp/pgl, obtidos para o Latossolo, pel os respectivos parâmetros da Areia Quartzosa Marinha. Obteve-se, com isso, uma série de razões adimensionais (Quadros 5 e 6). A figura 4 também mostra essas relações, mas de forma generalizada, isto é, sem identificação da

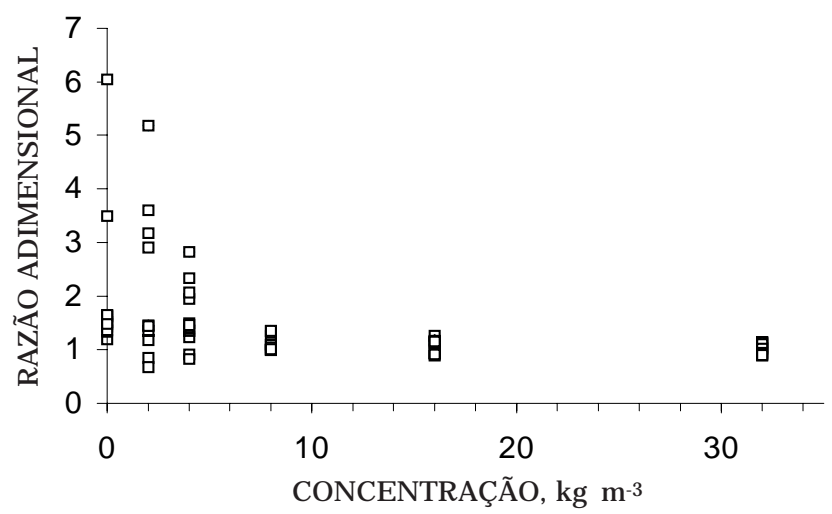

Figura 4. Relação adimensional dos parâmetros indicados nos quadros 5 e 6 em função da concentração de polímeros. variável considerada. Observa-se que, a partir da concentração de $8 \mathrm{~kg} \mathrm{~m}^{-3}$, os val ores dessas rel ações tornam-se aproximadamente constantes, tendendo para a unidade. Isso mostra, com efeito, que, para concentrações de polímeros acima de $8 \mathrm{~kg} \mathrm{~m}^{-3}$, as propriedades físico-hídricas dos meios porosos já não mais expressam seus efeitos naturais porque são dominadas pelas propriedades dos polímeros.

\section{CONCLUSÕES}

1. A capaci dade de retenção de água no L atossolo, para a concentração de $32 \mathrm{~kg} \mathrm{~m}^{-3}$, foi acrescida cerca de duas vezes, enquanto, na Areia Quartzosa Marinha, esse valor foi de 7,5 vezes.

2. Os parâmetros $\theta_{r}, \theta_{s}$ e a aumentaram com o aumento da concentração de polímeros, enquanto os valores da condutividade hidráulica decresceram progressivamente.

3. Os valores dos diâmetros de poros que armazenam mais água foram bem superiores quando da incorporação de polímeros, para os dois meios porosos, chegando a aumentar o diâmetro em até quatro vezes.

4. Os perfis de umidade resultantes da simulação do processo deevaporação da água para uma demanda de $5 \mathrm{~mm} / \mathrm{d}$, por $168 \mathrm{~h}$, demonstraram que a maior concentração testada duplicou a capacidade de armazenamento deágua no Latossolo, enquanto, na Areia Quartzosa Marinha, este aumento foi de 7,5 vezes.

5. De modo geral, a partir das concentrações de $8 \mathrm{~kg} \mathrm{~m}^{-3}$, as propriedades físico-hídricas dos dois meios porosos foram dominadas pelo efeito dos polímeros.

\section{CONSIDERAÇÕES FINAIS}

Os efeitos de polímer os hidrorretentores em sol os agrícol as ainda suscitam perguntas que necessitam ser respondidas, tais como:

1. Quando hidratados, os polímeros apresentam uma capacidade de retenção, em peso, de até 200 vezes, ou mais. No entanto, se primeiramente forem incorporados aos sol os e só então hidratados, esse aumento será na ordem de poucas vezes. Isso pode comprometer a disponibilidade de água às plantas?

2. A redução na condutividade hidráulica saturada dos sol os que recebem polímeros, aliada à capacidade de expansão dos hidrogéis, quando hidratados, pode facilitar o processo erosivo? 


\section{LITERATURA CITADA}

AZZAM, R.A.I . Polymeric conditioner gels for desert soils. Comm Soil Sci. Plant, 14:739-760, 1983.

BAASIRI, M.; RYAN, J .; MUCHEIK, M. \& HARIK, S.N. Soil application of a hydrophilic conditioner in relation to moisture, irrigation frequency and crop growth. Soil Sci. Plant, 17:573-589, 1986.

BLODGETT, A.M.; BEATTIE, D.J .; WHITE, J.W. \& ELLIOTT, G.C. Hydrophilic polymers and wetting agents affect absorption and evaporative water loss. J . Am. Soc. Hort. Sci., 28:633-635, 1993.

BORATTO, F. BASIC para engenheiros e cientistas. 2.ed. Rio de J aneiro, Livros Técnicos e Científicos, 1984. 120p.

FLANNERY, R.L. \& BUSSCHER, W.J . Use of a synthetic polymer in potting soils to improve water holding capacity. Soil Sci. Plant, 13:103- 111, 1982.
FONTENO, W.C. \& BILDERBACK, T.E. I mpact of hydrogel on physical properties of coarse-structured horticultural substrates. J . Am. Soc. Hort. Sci., 118:217-222, 1993.

PREVEDELLO, C.L. Física do solo com problemas resolvidos. Curitiba, Salesward-Discovery, 1996. 446p.

TAYEL, M.Y.; ABDED F.M. \& EL-HADY, O.A. Effect of soil condicioners on plantgrowth and water use efficiency ( $A$ green house experiment). Hort. Acta, 119:223-229, 1981.

van GENUCHTEN, M. Th. A closed-form equation for predicting hidraulic conductivity of unsaturated soil. Soil Sci. Soc. Am., 44:892-898, 1980.

VOLKMAR, K. M.\& CHANG, C. Influence of hydrophilic gel polymers on water relation and growth and yield of barley and canola. J . Can. Sci. Plant, 35:605-611, 1995.

WANG, Y.T. \& GREGG, L.L. Hydrophilic polymers - their response to soil amendments and effect on properties of a soilless potting mix. J. Am. Soc. Hort. Sci., 115:943-948, 1990. 